\title{
Elucidation of a protein-protein interaction network involved in Corynebacterium glutamicum cell wall biosynthesis as determined by bacterial two-hybrid analysis
}

\author{
Monika Jankute • Charlotte V. Byng • \\ Luke J. Alderwick • Gurdyal S. Besra
}

Published online: 13 August 2014

(C) The Author(s) 2014. This article is published with open access at Springerlink.com

\begin{abstract}
Mycobacterium species have a highly complex and unique cell wall that consists of a large macromolecular structure termed the mycolyl-arabinogalactan-peptidoglycan (mAGP) complex. This complex is essential for growth, survival and virulence of the human pathogen Mycobacterium tuberculosis, and is the target of several anti-tubercular drugs. The closely related species Corynebacterium glutamicum has proven useful in the study of orthologous M. tuberculosis genes and proteins involved in mAGP synthesis. This study examines the construction of a protein-protein interaction network for the major cell wall component arabinogalactan in C. glutamicum based on the use of a bacterial two-hybrid system. We have identified twenty-four putative homotypic and heterotypic protein interactions in vivo. Our results demonstrate an association between glycosyltransferases, GlfT1 and $\mathrm{AftB}$, and interaction between the sub-units of decaprenylphosphoribose epimerase, DprE1 and DprE2. These analyses have also shown that AftB interacts with AftA, which catalyzes the addition of the first three arabinose units onto the galactan chain. Both AftA and AftB associate with other arabinofuranosyltransferases, including Emb and $\mathrm{AftC}$, that elongate and branch the arabinan domain. Moreover, a number of proteins involved in arabinogalactan biosynthesis were shown to form dimers or multimers. These findings provide a useful recourse for understanding the biosynthesis and function of the mycobacterial cell wall, as well as providing new therapeutic targets.
\end{abstract}

Electronic supplementary material The online version of this article (doi:10.1007/s10719-014-9549-3) contains supplementary material, which is available to authorized users.

M. Jankute $\cdot$ C. V. Byng $\cdot$ L. J. Alderwick $\cdot$ G. S. Besra $(\bowtie)$

School of Biosciences, University of Birmingham, Edgbaston,

Birmingham B15 2TT, UK

e-mail: g.besra@bham.ac.uk
Keywords Arabinogalactan $\cdot$ Biosynthesis $\cdot$ Cell wall Corynebacteria $\cdot$ Mycobacteria $\cdot$ Protein-protein interactions

$\begin{array}{ll}\text { Abbreviations } \\ \text { AG } & \text { arabinogalactan } \\ \text { Araf } & \text { arabinofuranosyl } \\ \text { ArafT } & \text { arabinofuranosyltransferase } \\ \text { BACTH } & \text { bacterial adenylate cyclase two-hybrid } \\ \text { DP } & \text { decaprenyl phosphate } \\ \text { DPA } & \text { decaprenylphosphoryl-D-arabinofuranose } \\ \text { DPPR } & \text { decaprenylphosphoryl-5-phosphoribose } \\ \text { DPR } & \text { decaprenyl-5-phosphoribose } \\ \text { Gal } f & \text { galactofuranosyl } \\ \text { Gal } f \text { T } & \text { galactofuranosyltransferase } \\ \text { mAGP } & \text { mycolyl-arabinogalactan-peptidoglycan } \\ \text { PG } & \text { peptidoglycan } \\ \text { TB } & \text { tuberculosis }\end{array}$

\section{Introduction}

Mycobacterium tuberculosis the causative agent of tuberculosis (TB), remains a major cause of mortality and morbidity from a single infectious organism. In 2012, approximately 8.6 million people developed TB and 1.3 million died from the disease [1]. Emergence of multidrug-resistant [2], extensively drug resistant [2] and recently reported totally drug-resistant [3-5] clinical isolates has prompted the need for new drugs and drug targets. M. tuberculosis and other bacteria in the suborder of Corynebacterineae are characterized by a highly complex cell envelope. This cell wall is comprised of a cross-linked peptidoglycan (PG), covalently linked to arabinogalactan (AG) chains, which are further esterified by mycolic acids [6-8]. This macromolecular structure is often 
referred to as the mycolyl-arabinogalactan-peptidoglycan (mAGP) complex [9].

AG is composed predominantly of arabinofuranosyl (Araf) and galactofuranosyl (Galf) residues [10] and is covalently attached to PG via a specialized linker unit, L-Rhap- $(1 \rightarrow$ 4)- $\alpha$-D-GlcNAc [7]. The galactan domain of AG is composed of approximately 30 alternating $\beta(1 \rightarrow 5)$ and $\beta(1 \rightarrow 6)$ Gal $f$ residues connected in a linear fashion [11]. Three similar Darabinan chains comprising roughly $30 \mathrm{Araf}$ residues each are attached to the galactan chain [12]. Since the AG structure is essential to $M$. tuberculosis, many gene deletion studies investigating $A G$ have been performed in the closely related Corynebacterium genus, where aspects of AG biosynthesis are non-essential. Deletion studies in C. glutamicum demonstrated that the arabinan chains of AG are attached distinctively to the 8th, 10th, and 12th residue of the linear galactan chain [12]. Unlike most bacterial polysaccharides, AG lacks repeating units and is composed of a few distinct structural motifs, notably the terminal $\mathrm{Ara}_{6}$ motif, with the 5-OH of the t-Araf and 2-Araf residues representing sites for mycolylation $[11,8]$. Collectively, AG, PG, and mycolic acids with additional outer layer lipids result in an exceptionally robust and hydrophobic cell wall structure. Importantly, a number of anti-TB drugs, such as ethambutol [13-15] and isoniazid [16, 17], target the biosynthesis of the mAGP complex.

The biosynthesis of $A G$ involves the formation of the linkage unit synthesized on a decaprenyl phosphate lipid carrier (DP). Firstly, WecA transfers GlcNAc-1-P from the substrate UDP-GlcNAc-1-P onto the DP carrier [18, 19]. The rhamnosyltransferase WbbL then attaches the rhamnosyl residue to the DP-P-P-GlcNAc forming the full linker unit of AG, DP-P-P-GlcNAc-Rha $[18,20]$. The linker unit serves as an acceptor for the sequential addition of roughly $30 \mathrm{Gal} f$ residues. Bifunctional galactofuranosyltransferase (Galf $\mathrm{T}$ ) GlfT1 recognizes the linkage unit and transfers two Galf residues to DP-P-P-GlcNAc-Rha yielding DP-P-P-GlcNAc-Rha-Gal $f_{2}$ [21]. GlfT2 then attaches further Gal $f$ residues acting both as a UDP-Galf: $\beta$-D- $(1 \rightarrow 5)$ Gal $f$ T and a UDP-Galf: $\beta$-D-( $(1 \rightarrow 6)$ GalfT [22-24]. Arabinan biosynthesis employs decaprenylphosphoryl-D-arabinofuranose (DPA), the only known donor of Araf residues in AG biosynthesis. The assembly of DPA has been recently investigated in detail [25, 26]. DPA biogenesis begins with UbiA transferring 5phosphoribosyl-1-pyrophosphate to a DP forming decaprenylphosphoryl-5-phosphoribose (DPPR) [26]. DPPR is then dephosphorylated to decaprenyl-5-phosphoribose (DPR) by a putative phospholipid phosphatase [27]. DprE1 and DprE2 then catalyze the epimerization of DPR to DPA, consequently forming the essential sugar donor DPA [28-30]. A specialized arabinofuranosyltransferase (Araf $\mathrm{T}$ ) AftA transfers the first Araf residue from the substrate molecule DPA onto the 8th, 10th and 12th Galf residues of the galactan chain [12]. Further $\alpha(1 \rightarrow 5)$-linked Araf residues are added by
EmbA and EmbB in M. tuberculosis [31] or Emb in C. glutamicum [26]. Branching $\alpha(1 \rightarrow 3)$ Araf Ts, AftC and $\mathrm{AftD}$, are responsible for $\alpha(1 \rightarrow 3)$-linked Araf residues of the arabinan domain [32-35]. Finally, the terminal $\beta(1 \rightarrow 2)$ Araf residues are transferred from DPA onto the arabinan domain by AftB [36, 37].

The structure and biogenesis of AG has been fairly well described, however, certain aspects of its biosynthesis remain poorly understood. For instance, the characterization of multi-protein complexes has been extremely limited, perhaps due to a number of cell wall biosynthesis proteins being transmembrane or membrane bound. In this study, we attempted to investigate the associations between $C$. glutamicum proteins involved in the assembly of AG by using the bacterial adenylate cyclase two-hybrid (BACTH) system [38]. This system is based on the functional complementation between two fragments of the adenylate cyclase to restore a cAMP signaling cascade in Escherichia coli. Importantly, BACTH is able to detect physical interactions between both cytoplasmic as well as membrane proteins [39-43].

Our data supports interactions between various proteins involved in AG biosynthesis. Moreover, we demonstrate a number of novel interactions between these proteins. Altogether, these results suggest that proteins involved in AG assembly are associated to one another through multiple interactions.

\section{Materials and methods}

Bacterial strains and growth conditions

All cloning steps were performed in $E$. coli XL-1 Blue cells (Invitrogen). The E. coli cya strain BTH101 (( $\mathrm{F}^{-}$, cya-99, araD139, galE15, galK16, rpsL1 ( $\left.\mathrm{Str}^{\mathrm{r}}\right), h s d \mathrm{R} 2$, mcrA1, $m c r \mathrm{~B} 1)$ was used for the bacterial two-hybrid screen (Euromedex). E. coli strains were grown in Luria-Bertani (LB) medium at $30{ }^{\circ} \mathrm{C}$ or $37{ }^{\circ} \mathrm{C}$ as specified in the text. Plasmids were maintained with ampicillin $(100 \mu \mathrm{g} / \mathrm{ml})$ or kanamycin $(50 \mu \mathrm{g} / \mathrm{ml})$. LB agar reporter plates contained streptomycin $(100 \mu \mathrm{g} / \mathrm{ml})$, ampicillin $(100 \mu \mathrm{g} / \mathrm{ml})$, kanamycin (50 $\mathrm{\mu g} / \mathrm{ml})$, 5-bromo-4-chloro-3-indolyl- $\beta$-Dgalactopyranoside (X-gal; $40 \mu \mathrm{g} / \mathrm{ml}$ ) and isopropyl $\beta$-D-1thiogalactopyranoside (IPTG; $0.5 \mathrm{mM}$ ). MacConkey plates $\left(\right.$ Difco $\left.^{\mathrm{TM}}\right)$ contained streptomycin $(100 \mu \mathrm{g} / \mathrm{ml})$, ampicillin $(100 \mu \mathrm{g} / \mathrm{ml})$, kanamycin $(50 \mu \mathrm{g} / \mathrm{ml})$, IPTG $(0.5 \mu \mathrm{mM})$ and maltose (1\%). M63 minimal media plates [44] were supplemented with streptomycin $(50 \mu \mathrm{g} / \mathrm{ml})$, ampicillin $(50 \mu \mathrm{g} / \mathrm{ml})$, kanamycin $(25 \mu \mathrm{g} / \mathrm{ml}), X$-gal $(40 \mu \mathrm{g} / \mathrm{ml})$, IPTG $(0.5 \mathrm{mM})$ and maltose $(0.2 \%)$. 


\section{Plasmid construction}

All recombinant DNA methods were performed using standard protocols. Briefly, the genes involved in C. glutamicum AG biosynthesis were amplified from genomic DNA of C. glutamicum ATCC 13032. The plasmids have been constructed by inserting gene sequences of interest in pKT25 (T25 fusion at N-terminus), pKNT25 (T25 fusion at C-terminus), pUT18 (T18 fusion at C-terminus) and pUT18c (T18 fusion at N-terminus) [38], using oligonucleotides provided in Supplementary Table S1. The bacterial BACTH system kit was obtained from Euromedex and contained empty vectors together with positive control plasmids pKT25-zip and pUT18c-zip.

\section{Bacterial two-hybrid system}

Two plasmids expressing recombinant proteins bearing $\mathrm{N}$ - or C- terminal T25 and T18 fusions were co-transformed into E. coli BTH101 cells (Table S2). Cells were spread on LB plates containing streptomycin $(100 \mu \mathrm{g} / \mathrm{ml})$, ampicillin $(100 \mu \mathrm{g} / \mathrm{ml})$, kanamycin $(50 \mu \mathrm{g} / \mathrm{ml})$ and incubated at $30{ }^{\circ} \mathrm{C}$ for $48 \mathrm{~h}$. Several colonies were picked and used to inoculate $3 \mathrm{ml}$ of LB supplemented with appropriate antibiotics and $0.5 \mathrm{mM}$ IPTG. Cultures were grown overnight at $30{ }^{\circ} \mathrm{C}$ with shaking. Overnight cultures were washed three times in minimal media and spotted $(2 \mu \mathrm{l})$ onto LB, MacConkey or M63 minimal media agar plates supplemented with appropriate antibiotics and nutrients. The $\beta$-galactosidase assay was performed as described elsewhere [44]. The values presented are the mean of 3 independent activity assays.

\section{Statistical analysis}

The results are expressed as the means \pm S.D. and were analyzed using a Student's $t$-test to determine significant differences $(p<0.01)$ between samples.

\section{Results and discussion}

Network analysis of AG biosynthetic proteins

We initially aimed to identify whether any of the proteins involved in cell wall assembly have been predicted or demonstrated to make a functional network. Focusing on the list of proteins associated with AG biosynthesis we used the STRING database of interactions [45] to reveal a putative protein association network, with GlfT2 chosen as the network node (Fig. 1). The interaction patterns of proteins had a high confidence score $(>0.7)$ and served as a basis for selection of $C$. glutamicum proteins that were further analyzed using the in vivo BACTH system. The generated network contained ABC family transporters (RfbD and RfbE), GT-A type GalfTs GlfT1, and proteins involved in rhamnose sugar donor formation, all centered on GlfT2. Transmembrane Araf $\mathrm{T}$ Emb showed a strong evidence for interaction with AftA and AftB, as well as the uncharacterized protein NCg12596. The network also contained a putative phospholipid phosphatase NCg12782 and proteins involved in DPA synthesis: DprE1, DprE2 and UbiA.

\section{Bacterial two-hybrid analysis of AG proteins}

To characterize the physical interactions between components of the $C$. glutamicum cell wall biosynthetic machinery, the following full-length proteins WecA, WbbL, GlfT1, GlfT2, AftA, AftB, AftC, AftD, Emb, UbiA, DprE1, and DprE2, were tested systematically for pair-wise interactions using BACTH. The known or predicted function of proteins is shown in Table 1. Each protein was fused to the fragment of the catalytic domain of chimeric adenylate cyclase (T25 or T18) of Bordetella pertussis at either the C- or N-terminus (Table S2). Interaction between two hybrid proteins leads to reconstitution of the fragments of adenylate cyclase resulting in restoration of cAMP production in a E. coli cya mutant [38]. The resulting cAMP forms a complex with the catabolite activator protein and binds to various promoters, thus regulating transcription of several genes, including the lactose and maltose operons. The activation of these operons can be detected on selective agar plates or using a $\beta$-galactosidase assay. Importantly, this bacterial two-hybrid system was shown to be suitable to detect interactions between cytoplasmic, as well as transmembrane or membrane associated proteins [46, 47].

Despite several attempts, we did not succeed in constructing pKT25 and pUT18 derivatives expressing UbiA and AftD proteins, respectively. This is probably due to the toxicity of hybrid proteins to bacterial cells when expressed at high levels, which is especially true of membrane proteins. Moreover, the UbiA-T1 $8^{\mathrm{N}}$ and UbiA-T18 ${ }^{\mathrm{C}}$ hybrid proteins, when co-expressed with several other hybrid proteins, appeared to reduce down bacterial cell growth suggesting that overproduction of UbiA is toxic to $E$. coli cells.

To examine putative interactions between the hybrid proteins, $E$. coli BTH101 cells were co-transformed with pairs of recombinant plasmids (Table S2). In total, 577 pairs were screened for protein-protein interactions in vivo. All cotransformants, together with the positive and negative controls, containing either pKT25-zip/pUT18c-zip or empty pKT25/pUT18, were then spotted onto selective agar plates and the coloration of the colonies observed after $48 \mathrm{~h}$ of growth at $30^{\circ} \mathrm{C}$. In the absence of association between T25 and T18 fragments colonies appear white, whereas they are blue or red when functional complementation occurs. Representative 


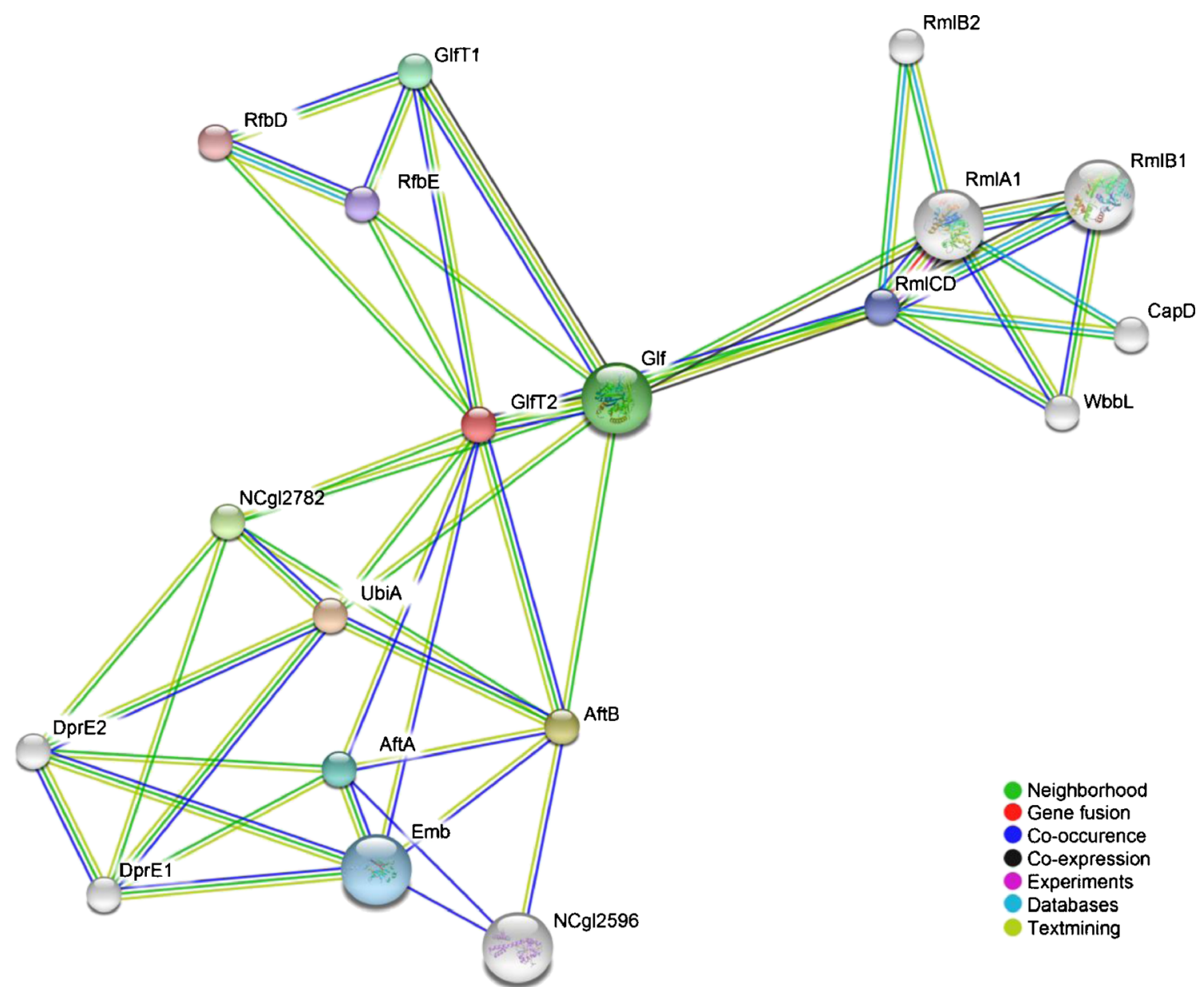

Fig. 1 Network of C. glutamicum proteins found to be important for cell wall assembly round the GlfT2 protein as determined by STRING analysis. Lines connecting the nodes indicate various interaction data supporting the network, colored by evidence type

Table 1 Predicted topology and function of C. glutamicum proteins described in this study

\begin{tabular}{lll}
\hline Protein & Predicted topology & Function \\
\hline WecA & transmembrane & UDP-GlcNAc-1-phosphatetransferase \\
WbbL & soluble & $\alpha$-3-L-rhamnosyltransferase \\
GlfT1 & soluble & UDP-galactofuranosyltransferase \\
GlfT2 & soluble & UDP-galactofuranosyltransferase \\
AftA & transmembrane & arabinofuranosyltransferase \\
AftB & transmembrane & arabinofuranosyltransferase \\
AftC & transmembrane & arabinofuranosyltransferase \\
AftD & transmembrane & arabinofuranosyltransferase \\
Emb & transmembrane & arabinofuranosyltransferase \\
DprE1 & soluble & decaprenylphosphoryl- $\alpha$-D-ribose \\
& & 2'-oxidase \\
DprE2 & soluble & decaprenylphosphoryl-D-2-keto erythro \\
& & pentose reductase \\
UbiA & transmembrane & decaprenyl-phosphate \\
& & 5-phosphoribosyltransferase \\
\hline
\end{tabular}

plates from the screening are shown in Fig. 2 and Supplementary Fig. S1-S13. Efficiency of the functional complementation between $\mathrm{T} 25$ and T18 domains was quantified by measuring $\beta$-galactosidase activity (Fig. S1-S13). Ultimately, 50 pairs of hybrid proteins resulted in a positive signal representing 24 putative homotypic and heterotypic proteinprotein interactions. The interaction results from the assays are summarized in Table 2.

Self-association of $C$. glutamicum cell wall biosynthesis proteins

Among all the tested proteins, dimerization or multimerization of WecA, GlfT1, GlfT2, AftA, AftB, AftC, DprE1, and DprE2 have been demonstrated employing BACTH. Co-expression of transmembrane WecA-T $25^{\mathrm{C}}$ and WecA-T $18^{\mathrm{C}}$ hybrid proteins restored a $\mathrm{cya}^{+}$phenotype and synthesis of CAMP in the E. coli cells, resulting in blue and red colonies on LB/M63Xgal and MacConkey media, respectively (Fig. S1a-c). The $\beta$-galactosidase assay revealed a significant increase in $\beta$ galactosidase activity ( $487 \pm 47$ Miller units) when compared 
a

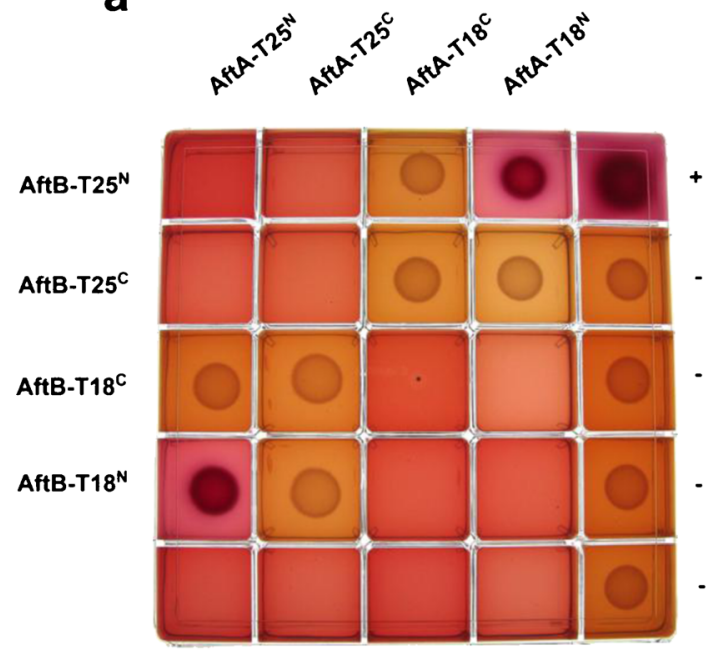

Fig. 2 BACTH analysis of interactions between AftA and AftB proteins from $C$. glutamicum. The genes encoding full-length proteins were fused in frame with adenylate cyclase $\mathrm{T} 25$ or T18 fragments at $\mathrm{N}$ - or C-terminus and expressed in E. coli cya ${ }^{-}$BTH101. a Co-transformants containing two plasmids encoding putative interaction partners were spotted onto selective MacConkey agar supplemented with appropriate antibiotics, $0.5 \mathrm{mM}$ IPTG and $1 \%$ maltose. Plates were incubated at $30{ }^{\circ} \mathrm{C}$ for $48 \mathrm{~h}$. Protein-protein interactions are indicated by red colonies through the reconstitution of adenylate cyclase catalytic domain. A strain coexpressing T25 and T18 fragments fused to leucine zipper domain was

to the negative control ( $86 \pm 11$ Miller units), containing empty pKT25, pKNT25, pUT18, and pUT18c plasmids (Fig. S1d). Importantly, the transmembrane fusions have to be correctly inserted into the plasma membrane with the T18 and T25 domains facing the cytoplasm in order an interaction to be detected. Therefore, suggesting that the $\mathrm{C}$ - terminus of GlcNAc-1-phosphate transferase WecA is cytoplasmic. This b

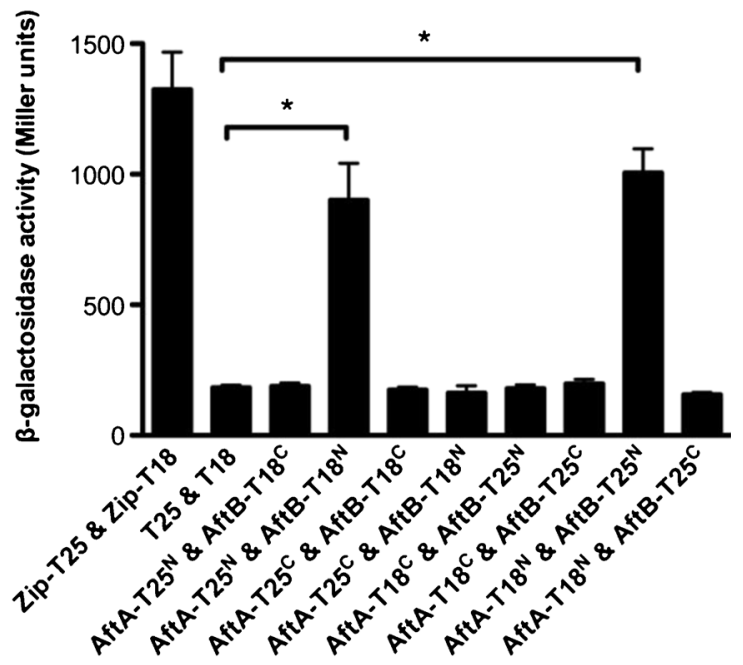

used as positive control $(+)$, whereas empty pKT25-pUT18, pKT25pUT18c, pKNT25-pUT18, and pKNT25-pUT18c were used as negative controls $(-)$. b The efficiencies of functional complementation between hybrid proteins were quantified by measuring $\beta$-galactosidase activities in suspensions of toluene treated E. coli BTH101 harboring the corresponding plasmids. Results are expressed in Miller units and are the mean \pm standard deviation of at least three independent experiments. Statistical significance was determined by Student's $t$-test $(\mathrm{p}<0.01)$

is in agreement with the predicted topology of WecA [48]. Physical self-dimerization or multimerization in vivo was also demonstrated for GlfT1 and GlfT2. Consistently, GlfT1-T2 $5^{\mathrm{N}}$ and GlfT1-T18 ${ }^{\mathrm{C}}$, GlfT1-T25 ${ }^{\mathrm{N}}$ and GlfT1-T18 ${ }^{\mathrm{N}}$, GlfT1-T25 ${ }^{\mathrm{C}}$ and GlfT1-T18 ${ }^{\mathrm{N}}$, GlfT2-T2 $5^{\mathrm{N}}$ and GlfT2-T18 ${ }^{\mathrm{C}}$, and GlfT2$\mathrm{T} 25^{\mathrm{C}}$ and GlfT2-T18 ${ }^{\mathrm{N}}$ hybrids restored $\mathrm{lac}^{+}$and $\mathrm{mal}^{+}$phenotypes and resulted in significant $\beta$-galactosidase activity

Table 2 Protein-protein interactions between C. glutamicum AG biosynthetic proteins determined by BACTH

\begin{tabular}{|c|c|c|c|c|c|c|c|c|c|c|c|c|}
\hline & WecA & WbbL & GlfT1 & GlfT2 & AftA & AftB & AftC & AftD & UbiA & DprE1 & DprE2 & Emb \\
\hline WecA & $\checkmark$ & & & & & & & & & & & \\
\hline WbbL & - & - & & & & & & & & & & \\
\hline GlfT1 & - & - & $\checkmark$ & & & & & & & & & \\
\hline GlfT2 & - & - & - & $\checkmark$ & & & & & & & & \\
\hline AftA & - & - & - & - & $\checkmark$ & & & & & & & \\
\hline AftB & $\checkmark$ & - & $\checkmark$ & - & $\checkmark$ & $\checkmark$ & & & & & & \\
\hline AftC & $\checkmark$ & - & - & - & $\checkmark$ & $\checkmark$ & $\checkmark$ & & & & & \\
\hline AftD & - & - & - & - & - & - & - & - & & & & \\
\hline UbiA & $\checkmark$ & $\checkmark$ & - & - & $\checkmark$ & $\checkmark$ & $\checkmark$ & - & - & & & \\
\hline DprE1 & - & - & - & - & - & - & - & - & - & $\checkmark$ & & \\
\hline DprE2 & - & - & $\checkmark$ & - & $\checkmark$ & $\checkmark$ & $\checkmark$ & - & - & $\checkmark$ & $\checkmark$ & \\
\hline Emb & $\checkmark$ & - & - & - & $\checkmark$ & $\checkmark$ & - & - & - & - & - & - \\
\hline
\end{tabular}

The positive interaction is indicated as $(\checkmark)$, whereas the lack of interaction is marked as (-) 
ranging from $266 \pm 69$ to $679 \pm 118$ Miller units (Fig. S1e-h). Recently, the structure of the polymerizing GlfT2 orthologue in M. tuberculosis has been solved revealing its assembly as a homotetramer [23], thus supporting the results obtained in this BACTH study.

Transmembrane AftA, AftB and AftC, proteins also tested positive for self-association. Co-expression of AftA-T2 $25^{\mathrm{N}}$ and AftA-T $18^{\mathrm{N}}$, AftB-T2 $5^{\mathrm{N}}$ and AftB-T $18^{\mathrm{N}}$, AftC-T25 ${ }^{\mathrm{C}}$ and AftC-T $18^{\mathrm{C}}$ combinations yielded $\beta$-galactosidase activity of $714 \pm 92,1185 \pm 265$, and $398 \pm 23$ Miller units, respectively (Fig. S2). The C-terminal region of AftA and AftB is predicted to be directed towards the periplasm [37, 12], therefore the lack of interaction between fusion pairs carrying $\mathrm{C}$-terminal $\mathrm{T} 25$ or T18 fragment was expected. In addition, BACTH experiments propose that the $\mathrm{N}$ - termini of $\mathrm{AftA}$ and $\mathrm{AftB}$ are cytoplasmic. In contrast to AftA and AftB, AftC is characterized by the absence of a periplasmic $\mathrm{C}$ - terminal extension [33]. Hence it is unsurprising that multimerization of $\mathrm{AftC}$ is observed with the fusion proteins tagged at the $\mathrm{C}$ - terminus. Interestingly, no evidence for homodimerization could be obtained for Emb and AftD. Finally, DprE1 and DprE2, both involved in DPA synthesis, appeared positive for self-interaction. DprE1-T2 $5^{\mathrm{N}}$ and DprE1-T18 ${ }^{\mathrm{C}}$, DprE1-T25 ${ }^{\mathrm{C}}$ and DprE1-T1 $8^{\mathrm{N}}$ fusions, as well as all four pairs of hybrid proteins co-expressing DprE2 led to a strong lacZ induction (ranged between $291 \pm 33$ and 1156 \pm 54 Miller units), significantly exceeding the negative control (Fig. S3).
In vivo interaction network among $\mathrm{AG}$ proteins

Next, we examined the interactions between different proteins involved in AG biosynthesis. Our results indicate that in addition to homodimerization, WecA is also able to interact with multiple partners of AG biosynthesis (Table 2). WecA-T $18^{\mathrm{C}}$ hybrid, when co-expressed with AftB-T2 $5^{\mathrm{N}}$, AftC-T25 ${ }^{\mathrm{C}}, \mathrm{Emb}-\mathrm{T} 25^{\mathrm{N}}$, and UbiAT25 ${ }^{\mathrm{C}}$ yielded significant $\beta$-galactosidase activities $1082 \pm 268,1047 \pm 186,1346 \pm 217$, and $1018 \pm 137$ Miller units, respectively (Fig. S4-5). BACTH also revealed an interaction with the rhamnosyltransferase WbbL, when UbiA hybrids were used as the bait. Cotransformation of $\mathrm{WbbL}-\mathrm{T} 18^{\mathrm{C}}$ or $\mathrm{WbbL}-\mathrm{T} 18^{\mathrm{N}}$ together with UbiA-T25 ${ }^{\mathrm{C}}$ led to a restoration of cAMP cascade with $\beta$-galactosidase activities of $1094 \pm 93$ and $1195 \pm$ 78 Miller units, respectively (Fig. S6a-d). Our studies have demonstrated the physical interaction between GlfT1 and AftB (Fig. S6e-h), as well as the DprE2 involved in DPA formation (Fig. S7a-d). Recent studies reported the physical interaction between GlfT1 and Rv3789, a small multidrug resistance-like transporter [49]. Rv3789 was proposed to target and stabilize the membrane associated GlfT1 [49]. Further experiments demonstrated evidence for a physical interaction between UbiA and AftA-T25 ${ }^{\mathrm{N}}$ (387 \pm 22 Miller units) (Fig. S8), AftB-T25 $5^{\mathrm{N}}(1015 \pm 185$ Miller units) (Fig. S10) and AftC- $225^{\mathrm{C}}(755 \pm 118$ Miller untis) (Fig. S12), responsible for the biosynthesis of
Fig. 3 An interaction network of C. glutamicum proteins involved in AG biosynthesis generated using yEd graph editor software. The circular arrows indicate selfassociation

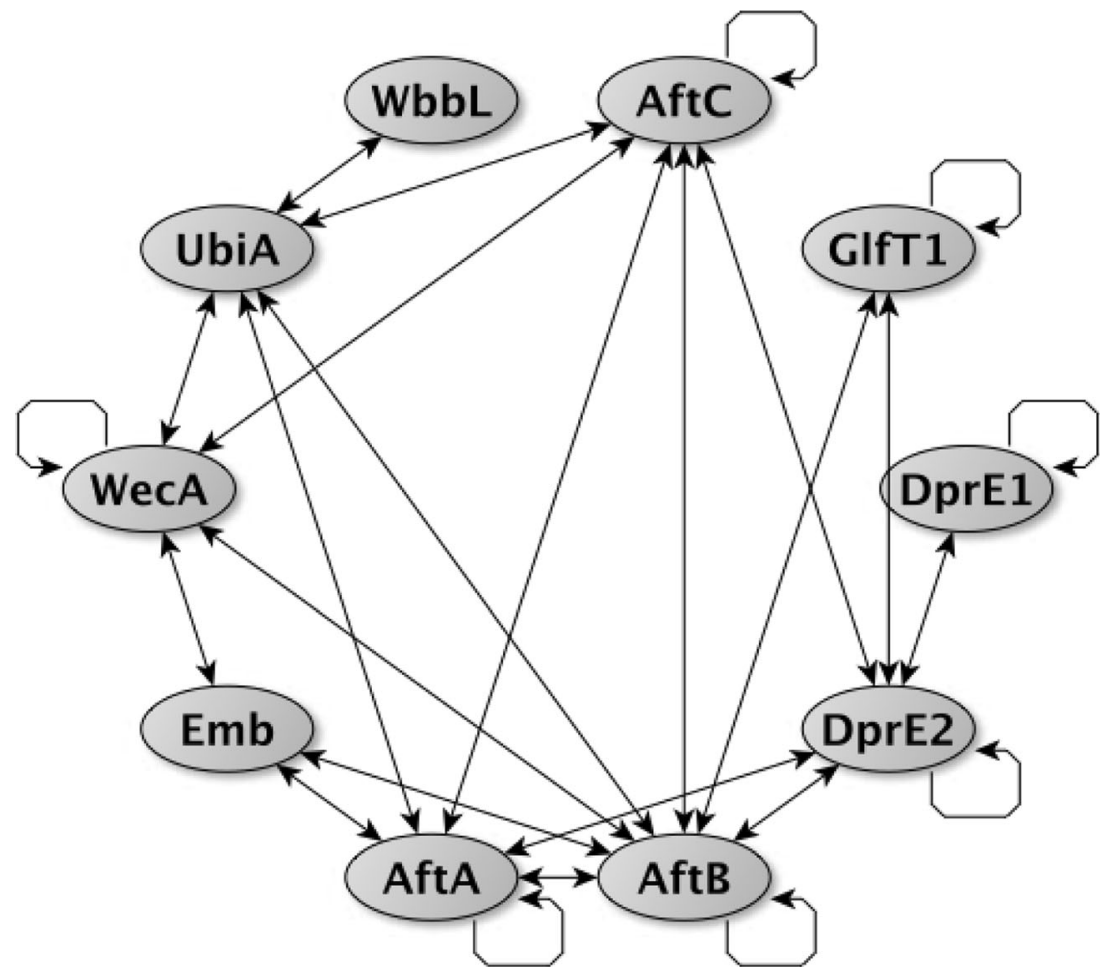


the arabinan domain of AG. Most of these ArafTs could also establish multiple interactions with each other. AftA, which primes the galactan chain of AG, associated with Emb, AftC and AftB (Fig. S7-9). In addition, AftB also interacted with Emb and AftC hybrid proteins (Fig. S10-11). Finally, C. glutamicum DprE1 was also found to strongly interact with DprE2. DprE1DprE2 association has been identified with seven different plasmid combinations resulting in a significant $\beta$-galactosidase activity ranging between $637 \pm 52$ and $1027 \pm 86$ Miller units (Fig. S13). Previous studies reported that orthologues of DprE1 and DprE2 in M. tuberculosis were able to catalyze the epimerization reaction in vitro, however, neither protein alone was sufficient to support this activity [49]. Thus, strongly suggesting that DprE1 and DprE2 work in concert to catalyze the conversion of DPR to DPA. However, when the same $M$. tuberculosis orthologues were experimentally tested for interaction using BACTH, co-transformants yielded negative results [49].

\section{Conclusions}

The majority of bacterial cell wall surface polysaccharides are built on a carrier lipid in the cytosolic side of the plasma membrane. Although it is not fully clear how and when these polymers are translocated to the periplasm, one could speculate that anchoring these macromolecules to the membrane positions them closely to the transporters and glycosyltransferases, therefore, promoting productive export across the plasma membrane. Formation of multi-protein complexes, that contain glycosyltransferases, enzymes forming its sugar nucleotides and transporters, is expected to be beneficial for the bacterial cell, since the tight arrangement of the biosynthetic reactions would retain productivity and accuracy of the polymerization process. We have demonstrated that the proteins responsible for the formation of the AG linker unit, WecA and WbbL, form a complex with decaprenylphosphoryl-5-phosphoribose synthase UbiA at the cytoplasmic membrane (Fig. 3). WecA and UbiA directly employ DP for the linker unit and DPPR formation, respectively $[18,30,50]$. Proximal interactions between WecA, WbbL and UbiA could perhaps facilitate synchronized utilization of DP for coordinated AG biosynthesis. In addition, UbiA show evidence for physical interaction between AftA, $\mathrm{AftB}$ and AftC proteins, which utilize DPA as a substrate (Fig. 3). It is possible that this multi-protein complex formation assists a mechanism similar to substrate channeling, where intermediary metabolic products of one enzyme are passed directly to another enzyme. Other DPA forming proteins, DprE1 and DprE2, showed evidence for a physical interaction. Interestingly, while both DprE1 and DprE2 are required for the epimerization reaction, there is evidence that C. glutamicum $\mathrm{NCgl1429}$ may play a similar function to DprE2 [28]. Investigation into potential DprE1-NCg11429 complexes could provide insight into this gene redundancy. Notably, GT-A glycosyltransferases GlfT1 and GlfT2 showed evidence for homodimerization using BACTH. GlfT1 transfers the first two Gal $f$ residues to the linker unit, whereas GlfT2 is responsible for addition of approximately $30 \mathrm{Gal} f$ residues in a linear chain. The recent crystal structure of M. tuberculosis GlfT2 in its apo-form and in complex with UDP, established its homotetrameric architecture [23]. Finally, AftA, AftB, AftC and Emb proteins involved in the assembly of arabinan domain in AG, indeed form a multi-protein complex at the inner membrane (Fig. 3). One could speculate that such a sophisticated complex would maintain the efficiency and fidelity of AG polymerization.

BACTH is a powerful technique for the investigation of protein-protein associations, however, several important notes should be highlighted regarding the significance of the interaction data obtained from BACTH. Firstly, the lack of lac $Z$ induction might be a result of plasmid instability, insoluble or dissipating fusions, and not the lack of direct physical interaction. Therefore, the hybrid proteins that test negative for interactions may still interact in vivo. Moreover, since the output of the interaction - cAMP - requires to be generated in the cytoplasm, these negative results may also result from the incorrect topological orientation of functional T25 and T18 domains into plasma membrane. In addition, using $\mathrm{BACTH}$, the fusion proteins are overexpressed when compared to the expression levels of native cells. Under these conditions, BACTH could have revealed a number of weak interactions between AG biosynthetic proteins. Although such associations would not take place at low protein concentrations, they can still occur when AG is being synthesized, where the local concentrations of proteins should be significantly higher. Finally, it is possible that some of the indentified interactions are a consequence of non-specific interactions initiated by endogenous $E$. coli host proteins that act as a tethering agent. These indirect associations, caused by a third protein, cannot be simply rejected.

In conclusion, our findings here suggest that enzymes involved in C. glutamicum cell wall assembly and precursor formation form complicated multi-protein complexes. We have identified 24 interactions in vivo between 12 proteins responsible for AG biosynthesis using BACTH. The challenge for the future will be to discover precisely how each of these multi-protein complexes form and function to synthesize and translocate AG.

Acknowledgments GSB acknowledges support in the form of a Personal Research Chair from Mr. James Bardrick, the Medical Research Council (MR/K012118/1), the Wellcome Trust (081569/Z/06/Z), and BBSRC. 
Open Access This article is distributed under the terms of the Creative Commons Attribution License which permits any use, distribution, and reproduction in any medium, provided the original author(s) and the source are credited.

\section{References}

1. WHO, T.: Global tuberculosis report 2012. World Health Organization, Geneva http://apps.who.int/iris/bitstream/10665/ 91355/1/9789241564656 eng.pdf (2012). Accessed 01 March 2014

2. WHO, T.: WHO progress report 2011: towards universal access to diagnosis and treatment of multidrug-resistant and extensively drugresistant tuberculosis. World Health Organization, Geneva http:// whqlibdoc.who.int/publications/2011/9789241501330_eng.pdf (2011). Accessed 01 March 2014

3. Velayati, A.A., Masjedi, M.R., Farnia, P., Tabarsi, P., Ghanavi, J., Ziazarifi, A.H., Hoffner, S.E.: Emergence of new forms of totally drug-resistant tuberculosis bacilli: super extensively drug-resistant tuberculosis or totally drug-resistant strains in iran. Chest 136(2), 420-425 (2009)

4. Udwadia, Z.F., Amale, R.A., Ajbani, K.K., Rodrigues, C.: Totally drug-resistant tuberculosis in India. Clin. Infect. Dis. 54(4), 579-581 (2012)

5. Migliori, G.B., De Iaco, G., Besozzi, G., Centis, R., Cirillo, D.M.: First tuberculosis cases in Italy resistant to all tested drugs. Euro Surveill 12 (5), E070517 070511 (2007).

6. Besra, G.S., Khoo, K.H., McNeil, M.R., Dell, A., Morris, H.R., Brennan, P.J.: A new interpretation of the structure of the mycolylarabinogalactan complex of Mycobacterium tuberculosis as revealed through characterization of oligoglycosylalditol fragments by fastatom bombardment mass spectrometry and ${ }^{1} \mathrm{H}$ nuclear magnetic resonance spectroscopy. Biochemistry 34(13), 4257-4266 (1995)

7. McNeil, M., Daffe, M., Brennan, P.J.: Evidence for the nature of the link between the arabinogalactan and peptidoglycan of mycobacterial cell walls. J. Biol. Chem. 265(30), 18200-18206 (1990)

8. McNeil, M., Daffe, M., Brennan, P.J.: Location of the mycolyl ester substituents in the cell walls of mycobacteria. J. Biol. Chem. 266(20), 13217-13223 (1991)

9. Jankute, M., Grover, S., Rana, A.K., Besra, G.S.: Arabinogalactan and lipoarabinomannan biosynthesis: structure, biogenesis and their potential as drug targets. Future Microbiol 7(1), 129-147 (2012)

10. McNeil, M., Wallner, S.J., Hunter, S.W., Brennan, P.J.: Demonstration that the galactosyl and arabinosyl residues in the cell-wall arabinogalactan of Mycobacterium leprae and Mycobacterium tuberculosis are furanoid. Carbohydr. Res. 166(2), 299-308 (1987)

11. Daffe, M., Brennan, P.J., McNeil, M.: Predominant structural features of the cell wall arabinogalactan of Mycobacterium tuberculosis as revealed through characterization of oligoglycosyl alditol fragments by gas chromatography/mass spectrometry and by ${ }^{1} \mathrm{H}$ and ${ }^{13} \mathrm{C}$ NMR analyses. J. Biol. Chem. 265(12), 6734-6743 (1990)

12. Alderwick, L.J., Seidel, M., Sahm, H., Besra, G.S., Eggeling, L.: Identification of a novel arabinofuranosyltransferase (AftA) involved in cell wall arabinan biosynthesis in Mycobacterium tuberculosis. J. Biol. Chem. 281(23), 15653-15661 (2006)

13. Takayama, K., Kilburn, J.O.: Inhibition of synthesis of arabinogalactan by ethambutol in Mycobacterium smegmatis. Antimicrob. Agents Chemother. 33(9), 1493-1499 (1989)

14. Belanger, A.E., Besra, G.S., Ford, M.E., Mikusova, K., Belisle, J.T., Brennan, P.J., Inamine, J.M.: The embAB genes of Mycobacterium avium encode an arabinosyl transferase involved in cell wall arabinan biosynthesis that is the target for the antimycobacterial drug ethambutol. Proc. Natl. Acad. Sci. U. S. A. 93(21), 11919-11924 (1996)
15. Telenti, A., Philipp, W.J., Sreevatsan, S., Bernasconi, C., Stockbauer, K.E., Wieles, B., Musser, J.M., Jacobs Jr., W.R.: The emb operon, a gene cluster of Mycobacterium tuberculosis involved in resistance to ethambutol. Nat. Med. 3(5), 567-570 (1997)

16. Winder, F.G., Collins, P.B.: Inhibition by isoniazid of synthesis of mycolic acids in Mycobacterium tuberculosis. J. Gen. Microbiol. 63(1), 41-48 (1970)

17. Banerjee, A., Dubnau, E., Quemard, A., Balasubramanian, V., Um, K.S., Wilson, T., Collins, D., de Lisle, G., Jacobs, W.R., Jr.: inhA, a gene encoding a target for isoniazid and ethionamide in Mycobacterium tuberculosis. Science 263 (5144), 227-230 (1994).

18. Mikusova, K., Mikus, M., Besra, G.S., Hancock, I., Brennan, P.J.: Biosynthesis of the linkage region of the mycobacterial cell wall. J. Biol. Chem. 271(13), 7820-7828 (1996)

19. Jin, Y., Xin, Y., Zhang, W., Ma, Y.: Mycobacterium tuberculosis Rv1302 and Mycobacterium smegmatis MSMEG 4947 have WecA function and MSMEG_4947 is required for the growth of M. smegmatis. FEMS Microbiol Lett 310 (1), 54-61 (2010).

20. Mills, J.A., Motichka, K., Jucker, M., Wu, H.P., Uhlik, B.C., Stern, R.J., Scherman, M.S., Vissa, V.D., Pan, F., Kundu, M., Ma, Y.F., McNeil, M.: Inactivation of the mycobacterial rhamnosyltransferase, which is needed for the formation of the arabinogalactanpeptidoglycan linker, leads to irreversible loss of viability. J. Biol. Chem. 279(42), 43540-43546 (2004)

21. Belanova, M., Dianiskova, P., Brennan, P.J., Completo, G.C., Rose, N.L., Lowary, T.L., Mikusova, K.: Galactosyl transferases in mycobacterial cell wall synthesis. J. Bacteriol. 190(3), 1141-1145 (2008)

22. Kremer, L., Dover, L.G., Morehouse, C., Hitchin, P., Everett, M., Morris, H.R., Dell, A., Brennan, P.J., McNeil, M.R., Flaherty, C., Duncan, K., Besra, G.S.: Galactan biosynthesis in Mycobacterium tuberculosis. Identification of a bifunctional UDPgalactofuranosyltransferase. J. Biol. Chem. 276(28), 26430-26440 (2001)

23. Wheatley, R.W., Zheng, R.B., Richards, M.R., Lowary, T.L., Ng, K.K.: Tetrameric structure of the GlfT2 galactofuranosyltransferase reveals a scaffold for the assembly of mycobacterial Arabinogalactan. J. Biol. Chem. 287(33), 28132-28143 (2012)

24. Poulin, M.B., Zhou, R., Lowary, T.L.: Synthetic UDPgalactofuranose analogs reveal critical enzyme-substrate interactions in GlfT2-catalyzed mycobacterial galactan assembly. Org. Biomol. Chem. 10(20), 4074-4087 (2012)

25. Alderwick, L.J., Lloyd, G.S., Lloyd, A.J., Lovering, A.L., Eggeling, L., Besra, G.S.: Biochemical characterization of the Mycobacterium tuberculosis phosphoribosyl-1-pyrophosphate synthetase. Glycobiology 21(4), 410-425 (2011)

26. Alderwick, L.J., Radmacher, E., Seidel, M., Gande, R., Hitchen, P.G., Morris, H.R., Dell, A., Sahm, H., Eggeling, L., Besra, G.S.: Deletion of $C g$-emb in corynebacterianeae leads to a novel truncated cell wall arabinogalactan, whereas inactivation of $\mathrm{Cg}$-ubiA results in an arabinan-deficient mutant with a cell wall galactan core. J. Biol. Chem. 280(37), 32362-32371 (2005)

27. Jiang, T., He, L., Zhan, Y., Zang, S., Ma, Y., Zhao, X., Zhang, C., Xin, Y.: The effect of MSMEG_6402 gene disruption on the cell wall structure of Mycobacterium smegmatis. Microb. Pathog. 51(3), $156-160(2011)$

28. Meniche, X., de Sousa-d'Auria, C., Van-der-Rest, B., Bhamidi, S., Huc, E., Huang, H., De Paepe, D., Tropis, M., McNeil, M., Daffe, M., Houssin, C.: Partial redundancy in the synthesis of the D-arabinose incorporated in the cell wall arabinan of Corynebacterineae. Microbiology 154(Pt 8), 2315-2326 (2008)

29. Crellin, P.K., Brammananth, R., Coppel, R.L.: Decaprenylphosphoryl-beta-D-ribose 2'-epimerase, the target of benzothiazinones and dinitrobenzamides, is an essential enzyme in Mycobacterium smegmatis. PLoS One 6(2), e16869 (2011)

30. Mikusova, K., Huang, H., Yagi, T., Holsters, M., Vereecke, D., D'Haeze, W., Scherman, M.S., Brennan, P.J., McNeil, M.R., Crick, 
D.C.: Decaprenylphosphoryl arabinofuranose, the donor of the Darabinofuranosyl residues of mycobacterial arabinan, is formed via a two-step epimerization of decaprenylphosphoryl ribose. J. Bacteriol. 187(23), 8020-8025 (2005)

31. Escuyer, V.E., Lety, M.A., Torrelles, J.B., Khoo, K.H., Tang, J.B., Rithner, C.D., Frehel, C., McNeil, M.R., Brennan, P.J., Chatterjee, D.: The role of the $e m b A$ and $e m b B$ gene products in the biosynthesis of the terminal hexaarabinofuranosyl motif of Mycobacterium smegmatis arabinogalactan. J. Biol. Chem. 276(52), 48854-48862 (2001)

32. Birch, H.L., Alderwick, L.J., Appelmelk, B.J., Maaskant, J., Bhatt, A., Singh, A., Nigou, J., Eggeling, L., Geurtsen, J., Besra, G.S.: A truncated lipoglycan from mycobacteria with altered immunological properties. Proc. Natl. Acad. Sci. U. S. A. 107(6), 2634-2639 (2010)

33. Birch, H.L., Alderwick, L.J., Bhatt, A., Rittmann, D., Krumbach, K., Singh, A., Bai, Y., Lowary, T.L., Eggeling, L., Besra, G.S.: Biosynthesis of mycobacterial arabinogalactan: identification of a novel alpha $(1 \rightarrow 3)$ arabinofuranosyltransferase. Mol. Microbiol. 69(5), 1191-1206 (2008)

34. Zhang, J., Angala, S.K., Pramanik, P.K., Li, K., Crick, D.C., Liav, A., Jozwiak, A., Swiezewska, E., Jackson, M., Chatterjee, D.: Reconstitution of functional mycobacterial arabinosyltransferase AftC proteoliposome and assessment of decaprenylphosphorylarabinose analogues as arabinofuranosyl donors. ACS Chem. Biol. 6(8), 819-828 (2011)

35. Skovierova, H., Larrouy-Maumus, G., Zhang, J., Kaur, D., Barilone, N., Kordulakova, J., Gilleron, M., Guadagnini, S., Belanova, M., Prevost, M.C., Gicquel, B., Puzo, G., Chatterjee, D., Brennan, P.J., Nigou, J., Jackson, M.: AftD, a novel essential arabinofuranosyltransferase from mycobacteria. Glycobiology 19(11), 1235-1247 (2009)

36. Belisle, J.T., Vissa, V.D., Sievert, T., Takayama, K., Brennan, P.J., Besra, G.S.: Role of the major antigen of Mycobacterium tuberculosis in cell wall biogenesis. Science 276(5317), 1420-1422 (1997)

37. Seidel, M., Alderwick, L.J., Birch, H.L., Sahm, H., Eggeling, L., Besra, G.S.: Identification of a novel arabinofuranosyltransferase AftB involved in a terminal step of cell wall arabinan biosynthesis in Corynebacterianeae, such as Corynebacterium glutamicum and Mycobacterium tuberculosis. J. Biol. Chem. 282(20), 14729-14740 (2007)

38. Karimova, G., Pidoux, J., Ullmann, A., Ladant, D.: A bacterial twohybrid system based on a reconstituted signal transduction pathway. Proc. Natl. Acad. Sci. U. S. A. 95(10), 5752-5756 (1998)

39. Karimova, G., Dautin, N., Ladant, D.: Interaction network among Escherichia coli membrane proteins involved in cell division as revealed by bacterial two-hybrid analysis. J. Bacteriol. 187(7), 2233-2243 (2005)

40. Karimova, G., Robichon, C., Ladant, D.: Characterization of YmgF, a 72-residue inner membrane protein that associates with the Escherichia coli cell division machinery. J. Bacteriol. 191(1), 333346 (2009)
41. Maxson, M.E., Darwin, A.J.: PspB and PspC of Yersinia enterocolitica are dual function proteins: regulators and effectors of the phage-shock-protein response. Mol. Microbiol. 59(5), 1610 1623 (2006)

42. Hara, Y., Seki, M., Matsuoka, S., Hara, H., Yamashita, A., Matsumoto, K.: Involvement of PlsX and the acyl-phosphate dependent sn-glycerol-3-phosphate acyltransferase PlsY in the initial stage of glycerolipid synthesis in Bacillus subtilis. Genes Genet. Syst. 83(6), 433-442 (2008)

43. Georgiadou, M., Castagnini, M., Karimova, G., Ladant, D., Pelicic, V.: Large-scale study of the interactions between proteins involved in type IV pilus biology in Neisseria meningitidis: characterization of a subcomplex involved in pilus assembly. Mol. Microbiol. 84(5), 857-873 (2012)

44. Battesti, A., Bouveret, E.: The bacterial two-hybrid system based on adenylate cyclase reconstitution in Escherichia coli. Methods 58(4), 325-334 (2012)

45. Szklarczyk, D., Franceschini, A., Kuhn, M., Simonovic, M., Roth, A., Minguez, P., Doerks, T., Stark, M., Muller, J., Bork, P., Jensen, L.J., von Mering, C.: The STRING database in 2011: functional interaction networks of proteins, globally integrated and scored. Nucleic Acids Res. 39(Database issue), D561-568 (2011)

46. Clarke, B.R., Greenfield, L.K., Bouwman, C., Whitfield, C.: Coordination of polymerization, chain termination, and export in assembly of the Escherichia coli lipopolysaccharide O9a antigen in an ATP-binding cassette transporter-dependent pathway. J. Biol. Chem. 284(44), 30662-30672 (2009)

47. Baulard, A.R., Gurcha, S.S., Engohang-Ndong, J., Gouffi, K., Locht, C., Besra, G.S.: In vivo interaction between the polyprenol phosphate mannose synthase Ppm1 and the integral membrane protein Ppm2 from Mycobacterium smegmatis revealed by a bacterial two-hybrid system. J. Biol. Chem. 278(4), 2242-2248 (2003)

48. Amer, A.O., Valvano, M.A.: Conserved amino acid residues found in a predicted cytosolic domain of the lipopolysaccharide biosynthetic protein WecA are implicated in the recognition of UDP-N-acetylglucosamine. Microbiology 147(Pt 11), 3015-3025 (2001)

49. Larrouy-Maumus, G., Skovierova, H., Dhouib, R., Angala, S.K., Zuberogoitia, S., Pham, H., Villela, A.D., Mikusova, K., Noguera, A., Gilleron, M., Valentinova, L., Kordulakova, J., Brennan, P.J., Puzo, G., Nigou, J., Jackson, M.: A small multidrug resistance-like transporter involved in the arabinosylation of arabinogalactan and lipoarabinomannan in mycobacteria. J. Biol. Chem. 287(47), 39933-39941 (2012)

50. Huang, H., Berg, S., Spencer, J.S., Vereecke, D., D'Haeze, W., Holsters, M., McNeil, M.R.: Identification of amino acids and domains required for catalytic activity of DPPR synthase, a cell wall biosynthetic enzyme of Mycobacterium tuberculosis. Microbiology 154(Pt 3), 736-743 (2008) 